\title{
Photoemission spectra of the $t-J$ model in one and two dimensions: similarities and differences
}

\author{
R. Eder ${ }^{1}$ and Y. Ohta ${ }^{2}$ \\ ${ }^{1}$ Department of Applied and Solid State Physics, University of Groningen, 9747 AG Groningen, The Netherlands \\ ${ }^{2}$ Department of Physics, Chiba University, Inage-ku, Chiba 263, Japan
}

(May 19, 2018)

\begin{abstract}
We present an exact diagonalization study of the single particle spectral function in the 1D and $2 \mathrm{D} t-J$ model. By studying the scaling properties with $J$ and $t$ we find a simple building pattern in $1 \mathrm{D}$ and show that every spectral feature can be uniquely assigned by a spinon and holon momentum. We find two types of low energy excitations: a band with energy scale $J$ and high spectral weight disperses upwards in the interior part of the Brillouin zone and reaches $E_{F}$ at $k_{F}$, and a band with energy scale $t$ and low spectral weight disperses downwards in the outer part of the zone, touching $E_{F}$ at $3 k_{F}$. An analogous analysis of the $2 \mathrm{D}$ case at half filling shows that the $t$-band exists also in this case, but is diffuse and never reaches the Fermi energy. For the doped case in $2 \mathrm{D}$ the picture is more reminiscent of $1 \mathrm{D}$, in particular the 'main-band' with a dispersion $\propto J$ and the 'shadow band' with energy scale $t$ can be identified also in this case. This leads us to propose that the shadow bands discovered by Aebi et al. in Bi2212 are the $2 \mathrm{D}$ analogue of the $3 k_{F}$ singularity in $1 \mathrm{D}$ systems and unrelated to antiferromagnetic spin fluctuations.
\end{abstract}

74.20.-Z, 75.10.Jm, 75.50.Ee

\section{INTRODUCTION}

Recent developments in photoelectron spectroscopy have challenged the apparent simple truth that the Fermi surface of cuprate superconductors is simply the one corresponding to LDA band structures with the only effect of the closeness to the Mott-Hubbard insulator being a moderate correlation narrowing of the band width. The discovery of the 'shadow bands' [1,2], the temperature dependent pseudogap in the underdoped state [3] and the substantial doping dependence of the quasiparticle band structure [1] leave little doubt that a simple single-particle description is quite fundamentally inadequate for these materials. Moreover, photoemission experiments on one-dimensional (1D) copper oxides [5] have shown very clear signatures of spin charge separation. The equally clear nonobservation of these signatures in the cuprate superconductors at any doping level advises against another apparent simple truth, namely that the Fermi surface seen in the cuprates is simply that of the 'spinons' in a 2D version of the Tomonaga-Luttinger liquid (TLL) realized in 1D. Motivated by these developments, we have performed a detailed exact diagonalization study of the electron removal spectrum in the 1D and $2 \mathrm{D} t-J$ model. This model reads

$$
H=-t \sum_{\langle i, j\rangle, \sigma}\left(\hat{c}_{i, \sigma}^{\dagger} \hat{c}_{j, \sigma}+\text { H.c. }\right)+J \sum_{\langle i, j\rangle}\left(\vec{S}_{i} \cdot \vec{S}_{j}-\frac{1}{4} n_{i} n_{j}\right) .
$$

There by the 'constrained' Fermion operators are written as $\hat{c}_{i, \sigma}=c_{i, \sigma} n_{i, \bar{\sigma}}$ and $\vec{S}_{i}$ denotes the spin operator on site $i$. The summation $\langle i, j\rangle$ extends over all pairs of nearest neighbors in a $1 \mathrm{D}$ or $2 \mathrm{D}$ square lattice.

The electron removal spectrum is defined as

$$
A(\vec{k}, \omega)=\frac{1}{\pi} \Im\left\langle\Psi_{0}\left|\hat{c}_{\vec{k}, \sigma}^{\dagger} \frac{1}{\omega-\left(E_{0}-H\right)-i 0^{+}} \hat{c}_{\vec{k}, \sigma}\right| \Psi_{0}\right\rangle,
$$

where $E_{0}$ and $\left|\Psi_{0}\right\rangle$ denote the ground state energy and wave function. For small finite clusters, this function can be evaluated numerically by means of the Lanczos algoritm [6].

In $1 \mathrm{D}$ the $t-J$ model is solvable by Bethe ansatz in the case $J=2 t$ [7], but even for this limit the complexity of the Bethe ansatz equations precludes an evaluation of dynamical correlation functions. For the closely related Hubbard model in the limit $J / t \rightarrow 0$ the Bethe-ansatz equations simplify $\mathbb{Q}$, and an actual calculation of the spectral function becomes possible [9, 10]. In all other cases Lanczos diagonalization is the only way to obtain accurate results for $A(\vec{k}, \omega)[11$.

In order to analyze our numerical results, we first want to develop an intuitive picture of the scaling properties of the elementary excitations in $1 \mathrm{D}$, which will turn out to be useful also in 2D. It has been shown by Ogata and Shiba [8] that for $J / t \rightarrow 0$ the wave functions can be constructed as products of a spinless Fermion wave function, which depends only on the positions of the holes, and a spin wave function, which depends only on the sequence of spins. A naive explanation for this remarkable property is the 'decay' of a hole created in a Néel ordered spin background into an uncharged spin-like domain wall, and a charged spinless domain wall. Then, since it is the kinetic energy $\sim t$ which propagates the charge-like domain walls, whereas the exchange energy $\sim J$ moves the spinlike domain walls, one may expect that the two types of domain walls have different energy scales. Namely the excitations of the charge part of the wave function (i.e., the 'holons') have $t$ as their energy scale, whereas those of 
the spin part (i.e., the 'spinons') have $J$ as their energy scale. Scanning the low energy excitation spectrum of 1D $t-J$ rings then shows that indeed most of the excited states have excitation energies of the form $a \cdot t+b \cdot J[12$, which indicates the presence of two different elementary excitations with different energy scales.

Surprisingly enough the low energy spectrum of the 2D model shows the same scaling behavior of the excitation energies as in 1D [12], which seems to indicate the existence of two types of spin and charge excitations if very different nature also in this case. Other cluster results indicate, however, that these two types of excitations do not exist as 'free particles': the dynamical density correlation function, which corresponds to the 'particle-hole excitations' of holons and shows sharp low energy peaks in 1D [7] is essentially incoherent in 2D and has practically no sharp low energy excitations [13]. The optical conductivity in 2D shows an incoherent high energy part with energy scale $J$ 14 - which is completely unexpected for the correlation function of the current operator which acts only on the charge degrees of freedom. There is moreover rather clear numerical evidence [15 17] that the hole-like low energy excitations can be described to very good approximation as spin 1/2 'spin bags' [18 i.e., holes dressed heavily by a local cloud of spin excitations.

To obtain further information about similarities and differences between $1 \mathrm{D}$ and 2D, also in comparison to the spectroscopic results, we have performed a systematic comparison of the electron removal spectra in both cases. As will become apparent, there are some similarities, but also clear differences. We suggest that the main difference between $1 \mathrm{D}$ and 2D is a strong attractive interaction between 'spinon' and 'holon' in 2D, which leads to a band of bound states being pulled out of the continuum of free spinon and holon states. This band of bound states which are nothing but simple spin 1/2 Fermions corresponding to the doped holes - then sets the stage for the low energy physics of the system, i.e., true spin-charge separation as in $1 \mathrm{D}$ never occurs.

\section{ONE DIMENSION, HALF FILLING}

We begin with a discussion of the $1 \mathrm{D}$ model at halffilling. Figure 1 shows the electron removal spectra for the 12-site ring. Let us first consider the left panel, where energies are measured in units of $J$. Then, one can distinguish different types of states according to their scaling behavior with $t$ : there is one 'band' of peaks (connected by the thin full line) whose energies relative to the single-hole ground state at $k=\pi / 2$ remains practically unchanged under a variation of $t$, i.e., these states have $J$ as their energy scale. As a remarkable fact, this 'band' abruptly disappears half-way in the Brillouin zone, i.e., there are no peaks whose energy scales with $J$ be- yond $k=\pi / 2$. This looks like a half-filled free-electron band with a Fermi level crossing at $\pi / 2$, which however is quite remarkable because inverse photoemission is not possible at half-filling. Next, in addition to this ' $J$-band', there are several groups of peaks whose excitation energy shows a very systematic progression with $t$. Indeed, when plotting the same spectra but measuring energies in units of $t$ (right panel of Fig. 11) these peaks coalesce, i.e., to excellent approximation the energy scale of these states is $t$. This coexistence of states with different energy scales can be nicely seen in the 'double peak' for $t / J=2$ and momentum $2 \pi / 6$ : the peak with lower binding energy falls into the $J$-band, the one with the higher binding energy belongs to the $t$-band. The dispersion of the $t$-band resembles a slightly asymmetric parabola with minimum near $\pi / 2$ for the low excitation energies that we are considering. The states that fall onto this parabola correspond to the creation of a spinon with momentum $k_{F}=-\pi / 2$, and a holon of momentum $k+\pi / 2$. Since the spinon momentum is fixed, this group of states then simply traces out the holon dispersion. On the other hand, the ' $J$-branch' corresponds to the holon momentum being fixed at the minimum of the holon dispersion, and thus traces out the spinon dispersion.

This building principle for the spectra can be pushed further. Namely, one might expect that not only $k_{F}$ but any spinon momentum may serve as the starting point for a complete branch of peaks which trace out the full holon dispersion. That this is indeed the case is shown in Fig. 22. There, the entire width of the spectra is shown and we have chosen the zero of energy at the excitation energy of either the topmost ' $J$-peak' at $k_{F}=\pi / 2$ (left panel) or the topmost ' $J$-peak' at $\pi / 3$ (right panel). Due to this choice of the zero of energy, the energy $\propto J$ of the spinon with the respective momentum drops out. Then, when measuring energies in units of $t$ different holon bands 'become sharp', i.e., their energy relative to the respective spinon energy scales accurately with $t$. Moreover, these different groups of peaks to good approximation all trace out the same simple backfolded nearest neighbor hopping dispersion, i.e., the dispersion of the holon is simply $2 t \cos \left(k_{x}\right)$. As discussed above, the first holon band is shifted by the spinons' Fermi momentum, $k_{F}=\pi / 2$, so that its dispersion near the band minimum at $k=\pi$ could be seen in Fig. 1. We have also verified that by alligning the spinon peaks at $\pi / 6$ yet another complete holon band can be identified.

We can thus infer the following building principle for the spectral function: the basis for the whole construction is the 'half-filled' spinon band, with dispersion $-0.65 J \cos (k)$; this is indicated by the thick dashed line in Fig. 3a. Then, each $(k, \omega)$-point of this band provides the 'basis' for a complete holon band $2 t \cos (k)$, which is 'hooked on' to the spinon band at its band maximum; these holon bands are indicated by the thin full lines in Fig. 3(a). Comparison with the numerical results (in this 
case for the 20-site ring) in Fig. 3(b) shows that indeed to excellent approximation the poles of the single particle spectral function fall onto these bands. There are some deviations at high binding energies, which however are most probably a deficiency of the Lanczos spectra, which are highly accurate only at low excitation energy. Moreover, the holon bands in Fig. 3(b) have been slightly shifted, i.e., they are 'hooked on' to the spinon band not precisely at their maximum - we have verified that this shift has oscillating sign for different chain lengths, so that it probably is a finite size effect. As an interesting feature, the pole strength seems to be constant along each of these holon bands, i.e., the weight is a function only of the spinon momentum (this seems not to be correct for $k=0$ and $k=\pi$; here it should be noted that for these momenta the holon band intersects itself, which leads to a doubling of the peak weight). In the thermodynamic limit, the density of bands increases, while simultaneously their spectral weight decreases, resulting in incoherent continua. Comparing with the exact results of Sorella and Parola [9] for the case $J / t \rightarrow 0$ it is obvious that the outermost holon band in our calculation, originating from the spinon Fermi momentum, develops into a cusp-like singularity of the spectral weight. The spinon band itself, whose energy scale is $J$, turns into a second dispersionless cusp in this limit, which skims at zero excitation energy between $k=0$ and $k=\pi / 2$. Sorella and Parola found the excitation energy of the dispersive cusp to be $-2 t \pm 2 t \sin (k)$, which corresponds to a the backfolded and shifted nearest neighbor hopping band, $-2 t+2 t \cos (k-\pi / 2)$.

Summarizing the data for 1D we see that the entire electron removal spectrum obeys a very simple building principle, which moreover holds for all momenta and frequencies. Analyzing the scaling of the different features with $J$ and $t$ one can identify 'branches' of states which trace out the dispersion of the true elementary excitations of the TLL, namely the collective spin and charge excitations. The dispersions of the spinons and holons are both consistent with simple nearest neighbor hopping bands, the spinons moreover have a half-filled Fermi surface. While these results may not be really new or surprising, we note that they demonstrate that exploiting the scaling properties of excitation energies provides a very useful method to identify the different 'subbands'. In the following, we will make extensive use of this principle to address the far less understood problems of 2D and finite doping.

\section{TWO DIMENSIONS, HALF FILLING}

We proceed to the $2 \mathrm{D}$ model, and also consider first the case of half filling. The spectra shown below refer to the standard 20-site cluster, which is the largest cluster for which the calculation of the electron removal spectrum is feasible also in the doped case. The $\vec{k}$-net for this cluster, which is shown in Fig. 田, consists of the group of momenta which roughly follows the $(1,1)$ direction, and a second group along $(\pi, 0) \rightarrow(0, \pi)$. We would like to stress that results for other clusters are completely consistent with those for the 20 -site cluster. Then, the left panel of Fig. 5 shows the photoemission spectrum for this cluster at half-filling; thereby we again focus on energies within a few $J$ from the top of the band and measure energies in units of $J$. When the spectra are aligned at the top of the band, the positions of the other dominant low energy peaks do not show a strong variation with $t$. Some peaks do show a slight but systematic drift with $t$, which however is much weaker than in $1 \mathrm{D}$. A peculiar feature is the peak at $(0,0)$, whose relative excitation energy decreases rather than increases with $t$. Inspection shows, however, that the (very weak) dispersion along the line $(\pi, 0) \rightarrow(0, \pi)$ (i.e., the lowest three momenta in Fig. E) scales with $t$ to good approximation. A possible explanation is the fact that a hole in a $2 \mathrm{D}$ system has two distinct mechanisms for propagation, firstly by 'string truncation', which gives effective hopping integrals $\sim J$, and secondly by hopping along spiral paths 19], which gives (smaller) effective hopping integrals $\sim t$ 20]. It can be shown [20] that the dispersion relation for a single hole to good approximation can be written as

$$
E(\vec{k})=J \cdot c_{1}\left(\cos \left(k_{x}\right)+\cos \left(k_{y}\right)\right)^{2}-t \cdot c_{2} \cos \left(k_{x}\right) \cos \left(k_{y}\right)
$$

where $c_{1} \gg c_{2}>0$ are numerical constants. The first term, which originates from the string truncation mechanism, gives a dispersion which is degenerate along $(\pi, 0) \rightarrow(0, \pi)$ and this degeneracy is lifted by the second term which is the contribution from the spiral paths; this naturally explains the scaling of the dispersion along this line with $t$. Comparing with 1D we note that with the exception of $(\pi, \pi)$ the ' $J$-band' is present in the entire Brillouin zone, i.e., the spinon Fermi surface seen at halffilling in 1D does not exist.

We turn to the right panel of Fig. 同(b), which shows the entire width of the spectra, with energies measured in units of $t$. It is first of all quite obvious that the spectra generally are more 'diffuse' than in 1D, with sharp features existing only in the immediate neighborhood of the top of the band (except for one relatively sharp high energy peak at $(\pi, \pi))$. Next, among the diffuse features at high energy there are some whose energy accurately scales with $t$. Although these 'peaks' are rather broad, so that the assignment of a dispersion is not really meaningful, their centers of gravity can be roughly fitted by the expression $-2 t \pm 2 t \sin \left(\left|k_{x}\right|+\left|k_{y}\right|\right)$, which is reminiscent of the dispersion of the 'holon-cusp' found by Sorella and Parola [9] in 1D. An important difference as compared to $1 \mathrm{D}$ is the fact that this $t$-band does not seem to reach the top of the photoemission spectrum - rather it stays an energy of $\sim t$ below the $J$-band, which forms the first ionization states. We believe that 'in 1D language' the 
most plausible interpretation of the data is the formation of bound states of spinon and holon: assuming a strong attraction between these two excitations, which may originate, e.g., from the well-known string mechanism for hole motion in an antiferromagnet [21], one may expect that a band of bound states is pulled out of the continuum of free spinons and holons. This band of bound states corresponds to the $J$-band (which however has a small contribution $\propto t$ in its dispersion due to the spiral path mechanism). Such a bound state of spinon and holon should be a spin-bag-like spin 1/2 Fermion, i.e., a hole heavily dressed by spin excitations. There is strong numerical evidence 15 17, that this is indeed the character of the low energy states in 2D at low doping. One may expect, however, that such a bound state may not be stable for all momenta, and we believe that this is the reason for the absence of a $J$-peak at $(\pi, \pi)$. In this picture, the $2 \mathrm{D}$ analogue of the holon is not a coherently propagating excitation, because it is bound to the much slower spinon by the linearly ascending string potential. This picture fits nicely with the diffuse character of the dynamical density correlation function in $2 \mathrm{D}$ [13]: this function, which in a TLL should measure basically the response of the free holons, in 2D has almost exclusively diffuse high energy 'peaks', with virtually no sharp low energy peaks. Moreover, the unexpected (in the framework of spin charge separation) appearance of $J$ as energy scale in the optical conductivity is also readily understood in terms of the dipole-excitations of a bound spinon-holon pair [14].

Summarizing the data for $2 \mathrm{D}$, we see a band of quasiparticle peaks, which predominantly has $J$ as its energy scale, and some diffuse high energy 'band' with energy scale $t$. Both, the absence of the 'spinon Fermi surface', as well as the lack of sharp 'holon bands' are in clear contrast to the situation in 1D. The formation of bound states of spinon and holon, resulting in a split-off band of spin-bag-like spin 1/2 Fermions explains this in a natural way.

\section{ONE DIMENSION, DOPED CASE}

We return to $1 \mathrm{D}$ and consider the doped case. Figure 6 shows the spectral function for the 12-site ring with 2 holes. Measuring excitation energies in units of $J$ (left panel) we can again identify the spinon band. For 2 holes in 12 sites the nominal Fermi momentum is $k_{F}=5 \pi / 12$ (i.e., half way between $\pi / 3$ and $\pi / 2$ ) and the spinon band extends up to this momentum. As was the case at halffilling, some other peaks show a systematic progression of their excitation energy, and switching the unit of energy to $t$ (right panel) again makes a nearly complete 'holon band' visible to which these peaks belong. The holon band again takes the form of a backfolded tight-binding band, but this time the top of the parabola around $k=\pi / 2$ is missing. The holon band now seems to touch the Fermi energy at $k_{F}$ and at $3 k_{F}=9 \pi / 12$ (the latter momentum is half way between $4 \pi / 6$ and $5 \pi / 6)$. This picture of the spectral function nicely fits with the recent exact calculation in the limit $J / t \rightarrow 0$ by Penc et al. [10]: on the photoemission side, this calculation showed a high intensity 'band' which is very similar to the backfolded tight-binding dispersion of the holon band. In addition there was a dispersionless low intensity band at zero excitation energy, which corresponds to the spinon band in the limit $J / t \rightarrow 0$. For both, the exact result in the limit $J / t \rightarrow 0^{+}$, and our numerical data for finite $J$, there are thus two branches of states which reach excitation energy zero: the 'main band' which touches $E_{F}$ at $k_{F}$, and the 'shadow band' [11], which reaches $E_{F}$ at $3 k_{F}$. The 'Fermi level crossings' of these two bands may be thought of producing the well known (marginal) singularities in the electron momentum distribution $n(k)$ at $k_{F}$ and $3 k_{F}$, found by Ogata and Shiba 8].

The numerical spectra demonstrate a peculiar feature of the TLL, namely a kind of Pauli exclusion principle which holds for both holons and spinons: the dispersions of both types of excitations become incomplete upon doping, i.e., the spinon Fermi surface shrinks as if the spinons were spin $1 / 2$ particles, while simultaneously the top of the holon band is 'sawed off' as if the holons were spinless Fermions. It should be noted that this is quite naturally to be expected in that the rapidities for the different 'particles' in the Bethe ansatz solution both obey a Pauli-like exclusion principle [7]. This has negative implications for, e.g., slave boson mean-field calculations, which necessarily have to treat one type of excitation as a Boson. While spin-charge separation is often quoted as justification for the mean-field decoupling, it is obvious that this approximation must fail to reproduce the excitation spectrum even qualitatively in $1 \mathrm{D}$, the only situation where spin-charge separation is really established.

For a more quantitative discussion of the Fermi points, we note that the Fermi momentum for hole concentration $\delta$ is $k_{F}=\frac{\pi}{2}(1-\delta)$. For this momentum the first branch of low energy excitations reaches $E_{F}$. For small $\delta$ the second branch of low energy excitations comes up to $E_{F}$ at $-3 k_{F}+2 \pi=\frac{\pi}{2}(1+3 \delta)$. The two marginal singularities thus enclose a hole pocket of length $2 \pi \delta$ as one would expect for holes corresponding to spinless Fermions. It is easy to see, that this hole pocket is nothing but the manifestation of the holon 'Fermi surface' around $k=\pi$ : the lowest charge excitations, which may be thought of as corresponding to a particle hole excitation between the two edges of the holon pocket have wave vector $4 k_{F}=2 \pi-2 \pi \delta$, i.e., the holon pocket has a diameter of $2 \pi / \delta$, precisely the distance between the two marginal singularities. The spectral function for the doped case thus follows the same building principle as for the case of half filling, with the sole difference being that occupied spinon or holon momenta are no longer avail- 
able for the construction of final states. The singularities in $n(k)$ may be thought of as enclosing a hole pocket corresponding to spinless Fermions, and thus reflect the Fermi surface of the holons. The two holon pockets are placed such that their inner edges at $\pm k_{F}$ enclose the volume corresponding to the Fermi sea of spinons of density $(1 / 2)(1-\delta)$

\section{TWO DIMENSIONS, DOPED CASE}

We proceed to the doped case in 2D. Let us note from the very beginning that for very simple technical reasons the situation is much more unfavorable in this case. To begin with, due to the higher symmetry of $2 \mathrm{D}$ clusters the available $\vec{k}$ meshes are much coarser: for example, amongst the 18 allowed momenta in the 18 site cluster only $6 \vec{k}$-points are actually non symmetry-equivalent, so that the amount of nonredundant information is much smaller than in 1D. Next, unlike 1D where a unique relationship exists between hole density and Fermi momentum, most electron numbers in small 2D clusters correspond to open-shell configurations with highly degenerate ground states for noninteracting particles. In an open-shell situation multiplet effects are guaranteed to occur, so that it is in general unpredictable which momenta are occupied and which ones are not (this holds for a Fermi liquid, but is most probably true also for other 'effective particles'). Unexpected problems may arise from this. Bearing this in mind, one therefore may not expect to see a similarly detailed and clear picture as in 1D.

Then, Figure 7 shows the photoemission spectra for the 18-site cluster, with two holes. We first consider the left hand panel, where energies are measured in units of $J$. Comparing with Fig. 6, some similarities are quite obvious: the excitation energies of the topmost peaks at $(0,0),(2 \pi / 3,0)$ are independent of $t$ (although the spectra for $t / J=2$ show a slight deviation) so that we can identify a 'band' of states with energy scale $J$. The situation actually is not entirely clear, in that the peak at $(\pi / 3, \pi / 3)$ is so close in energy to the one at $(2 \pi / 3,0)$ that it is not possible to decide if their energy difference scales with $J$ or $t$. Next, the topmost peaks at $(2 \pi / 3,2 \pi / 3)$ and $(\pi / 3, \pi)$ show a systematic progression with $t$, which is very reminiscent of, e.g., Fig. 11. Plotting the same spectra with energy scale $t$ indeed to good approximation aligns these peaks (although the peak at $(2 \pi / 3,2 \pi / 3)$ still has a slight drift), i.e., their excitation energy relative to the topmost peak at $(2 \pi / 3,0)$ scales with $t$. Moreover one can identify a number of diffuse 'features' at energies between $-0.5 t$ and $-t$, which also are roughly aligned; these are indicated by the dashed line. In analogy with 1D, we can thus distinguish different branches of states, with different energy scales in their excitation energies. While the coarseness of the $\vec{k}$ - meshes introduces some uncertainty, the data are consistent with a ' $J$-band' dispersing upwards in the interior of the antiferromagnetic Brillouin zone, and a ' $t$-band' dispersing downwards in the outer part, i.e., the same situation as seen in 1D. A major difference is the fact that the 'features' at higher binding energies are all very diffuse, at least for $J / t \geq 3$. More significantly, despite the fact that its energy scale seems to be $t$, the dispersion of the 'shadow band' is much weaker than in 1D. In other words, the effective mass of that band is $\sim t^{-1}$, but with a very large prefactor.

We proceed to the 20-site cluster, also doped with two holes (see Fig. 8). Choosing $J$ as the unit of energy, we see the already familiar situation: the topmost peaks for the states at $(2 \pi / 5, \pi / 5)$ and $(\pi / 5,3 \pi / 5)$ are aligned (although $t / J=2$ again deviates slightly) and several other peaks show a systematic progression with $t$ (an unexpected exception is $(0,0)$ where a well defined peak actually is not observed). Changing to energy scale $t$ aligns a number of these peaks, which suggests that these peaks form a ' $t$-band' which originates from the topmost peak at $(2 \pi / 5, \pi / 5)$. This is a second unexpected feature of the 20-site cluster, in that for the spectra in 1D (and for those of the 18-site cluster in 2D) the most intense $t$ band always seemed to originate from the topmost peak of the photoemission spectrum. We can only speculate that these unusual features are the consequence of, e.g., the multiplet effects mentioned above. We also note in this context that the spectra at $(0,0)$ look actually quite different for 18 and 20 site cluster, which shows the impact of finite-size effects.

Ascribing the special behavior at $(0,0)$ to finite sizeeffects, we have a quite similar picture as in the 18-site cluster, i.e., the topmost peaks for spectra inside the antiferromagnetic zone have $J$ as their energy scale whereas the topmost peaks in the outer part of the zone have energy scale $t$ (this also holds for $(\pi, 0)$ which is on the boundary of the antiferromagnetic zone). As was the case in the 18 site cluster the 'shadow band', while having $t$ as its energy scale, has a much weaker dispersion than in 1D. Indeed, fitting the $t$-bands in both 18 and 20 site cluster by an expression of the form $2 t_{e f f}\left(\cos \left(k_{x}\right)+\cos \left(k_{y}\right)\right)$ requires to choose $t_{\text {eff }} \approx 0.1 t$ - it is tempting to speculate that this may actually be $\delta t$, as one would expect e.g. in the Gutzwiller picture. Another notable feature is that the $t$-band is restricted to the outer part of the Brillouin zone. Only the diffuse high-energy 'band' indicated by the dashed line in Figure 8 seems to scale with $t$.

For completeness we would like to mention that a similar analysis was not possible for the 16-site cluster with 2 holes. The reason is essentially that for some momenta there are no more sharp 'peaks', but rather a multitude of densely spaced small peaks. Due to this, we were not able to assign any defined 'bands', or groups of peaks which showed a systematic scaling of their excitation energy. We have also performed this kind of analysis for 
the 16 site cluster with 4 holes and found no more indication of the energy scale $J$ : at this somewhat higher concentration the entire spectra scale with $t$.

Summarizing the data for $2 \mathrm{D}$, hole doping seems to lead to behavior which is more reminiscent of $1 \mathrm{D}$ than for half filling, in that the $J$-band dispersing upwards in the inner part of the Brillouin zone and the $t$-band dispersing downwards in the outer part seem to exist also in this case. Much unlike 1D, however, the shadow band, while in principle having $t$ as its energy scale, still has a very weak dispersion, so that the band structure in the doped case is practically identical to that in the undoped system [22]. We note however, that the fact that the shadow band has $t$ as energy scale has profound implications for its explanation: there have been attempts to interpret the shadow band in Bi2212 as a 'dynamical replica' of the main band, created by scattering of quasiparticles in the standard tight-binding band from antiferromagnetic spin fluctuations 23]. Experimentally, however, the fact that the shadow bands are observed also in the overdoped compounds [2], where antiferromagnetism is very weak, as well as the fact that they do not seem to become more pronounced in the underdoped compounds [4], where antiferromagnetism is strong, both suggest otherwise. On the theoretical side, we believe that our data very clearly rule out this interpretation: both the 'main band' and the spin correlation function $\sqrt{13}$ have $J$ as their relevant energy scale, and it would be very hard to understand how the energy scale of $t$ for the shadow band should emerge from a combination of these two types of excitations. In fact, the relatively accurate scaling with physically very different parameters suggests completely different propagation mechanisms for the two types of excitations. We therefore believe that the shadow band is a separate branch of excitations, probably best comparable to the states which produce the $3 k_{F}$ singularity in the $1 \mathrm{D}$ systems.

\section{DISCUSSION}

In the previous sections we have investigated the photoemission spectrum for the one and two dimensional $t-J$ model. By studying the parameter dependence of the spectra, we could in 1D identify 'branches' of states which trace out the dispersions of the elementary excitations of the TLL, the spinons and holons. Both elementary excitations have a simple nearest neighbor hopping dispersion, but with different band width: that of the spinons is $\sim J$, that of the holons $\sim t$.

In the doped case there are two groups of states which touch the Fermi energy (see Figure 9). 'Inside' the noninteracting Fermi surface, there is a whole continuum of bands dispersing upwards to $E_{F}$. The uppermost of these bands traces out the spinon dispersion and has $J$ as its energy scale, the lowermost band traces out the holon dispersion and has $t$ as its energy scale. In the thermodynamic limit these bands degenerate into 'cusps' and merge at $E_{F}$. In the outer half of the Brillouin zone, there are only states which have $t$ as their energy scale. These reach the Fermi energy at $3 k_{F}$, giving rise to a second Fermi point. While the resolution in $k$ and $\omega$ available in our finite clusters is not sufficient to make statements about extreme low energy excitations, the positions of the singularities in the electron momentum distribution as determined from exact solutions clearly shows that both branches of states indeed do touch $E_{F}$. The two singularities may be thought of enclosing a hole pocket of extent $2 \pi \delta$, which is essentially the image of the holon Fermi surface.

In 2D at half-filling, the situation is quite different: while it is still possible to distinguish bands with different scaling behavior with $J$ and $t$, the spinon Fermi surface present in 1D does not exist and the 'holons' seem to correspond to overdamped resonances rather than sharp excitations as in 1D. We propose that strong attraction between spin and charge excitations, most probably due to the well-known string mechanism, pulls a band of bound states out of the continuum of 'free' holon and spinon states. The relevant physics thus is that of spin-bag-like spin 1/2 quasiparticles, as suggested by a considerable amount of numerical evidence.

For the doped case in 2D, the situation is less clear and actually somewhat ambiguous. The numerical photoemission spectra show some analogy with $1 \mathrm{D}$, in that there seems to be a high intensity 'main band' with energy scale $J$ dispersing upwards in the inner part of the Brillouin zone, and a low intensity 'shadow band' with energy scale $t$ dispersing downwards in the outer part of the Brillouin zone (see Figure 9). In contrast to 1D the dispersion of the shadow band is much weaker, i.e., while the energy scale of the dispersion is $t$, it has an additional very small prefactor (of the order of the hole concentration). Moreover the $t$-band seems limited to the outer part of the Brillouin zone, i.e. there are no indications for a holon band with energy scale $t$ dispersing upwards in the inner part of the Brillouin zone. Only in the 18-site cluster a diffuse 'band' with energy scale $t$ can be roughly identified at higher binding energies. The different energy scales of main band and shadow band suggest that these are excitations of quite different nature, and in particular rule out the explanation that that the shadow band is created by scattering from antiferromagnetic spin fluctuations.

Turning to experiment, the results for 2D immediately suggests a comparison with the data of Aebi et al. [1]. These authors found that in addition to the 'bright' part of the band structure, which seems to be consistent with the noninteracting one, there is also a low intensity 'replica', shifted approximately by $(\pi, \pi)$, which had been consistently overlooked in all previous studies. If one wants to make a correspondence to the situation for 
the $t-J$ model, one thus should identify this low intensity part with the $t$-band dispersing downwards in the $t-J$ model. Our data imply that the shadow band should have a slightly different dispersion than the main band. The limitations of the cluster method probably preclude any meaningful quantitative statements, but it might be interesting to see if this difference in dispersion can be resolved experimentally.

We conclude by outlining a somewhat speculative scenario, based on the assumption that the two bands represent indeed different excitations, which persist at all temperatures and independent of antiferromagnetic correlations. In this case, the topology in $2 \mathrm{D}$ opens an interesting possibility: whereas in $1 \mathrm{D}$ the two classes of low energy excitations forming the $k_{F}$ and $3 k_{F}$ singularities in $n(\vec{k})$ are well separated in $\vec{k}$ and $\omega$ space for simple topological reasons, the experimental data of Aebi et al. indicate that the main and shadow band intersect at certain points in the Brillouin zone (see Fig. 10, left panel). Neglecting the small difference in dispersion between main and shadow band, we might therefore model the low energy excitation spectrum by the effective Hamiltonian

$$
H_{Q P}=\sum_{\vec{k}, \sigma}\left(\epsilon(\vec{k}) a_{\vec{k}, \sigma}^{\dagger} a_{\vec{k}, \sigma}+\epsilon(\vec{k}+\vec{Q}) b_{\vec{k}, \sigma}^{\dagger} b_{\vec{k}, \sigma}\right)
$$

where $\epsilon(\vec{k})$ is the dispersion of the main band, $\vec{Q}=$ $(\pi, \pi)$, and the $a$ and $b$ operators refer to the main and shadow band, respectively. Choosing a dispersion of the form $\epsilon(\vec{k})=-2 t\left(\cos \left(k_{x}\right)+\cos \left(k_{y}\right)\right)+4 t^{\prime} \cos \left(k_{x}\right) \cos \left(k_{y}\right)$ this Hamiltonian reproduces the Fermi surface topology found by Aebi et al. quite well (see Fig. 10). However, as mentioned above the two branches of excitations intersect at some points of the Brillouin zone, so that already a small mixing between the two bands, which in turn may originate from the spinon-holon interaction, has a dramatic effect on the topology of the low energy excitation spectrum. Namely adding a term of the form

$$
H_{m i x}=\Delta \sum_{\vec{k}, \sigma}\left(a_{\vec{k}, \sigma}^{\dagger} b_{\vec{k}, \sigma}+\text { H.c. }\right),
$$

i.e., a hybridization between the two types of bands, even relatively small values of $\Delta$ open up a gap around $(\pi, 0)$ and transform the Fermi surface transformed into a hole pocket (see left panel of Fig. 10). Thereby we fix the chemical potential by requiring that the number of $a$ and $b$ particles remains unchanged; it is easy to see that the area covered by the pockets then equals the hole concentration $\delta$, precisely as it was the case in 1D. Thereby the Fermi surface has predominant main band character at its inner edge, and shadow band character at the outer edge, implying a very different 'visibility' in photoemission. Finally, it is tempting to speculate that the 'pseudo gap order parameter' $\Delta$ decreases with increasing temperature/hole concentration. Its vanishing at a certain temperature $T^{*}$ then could produce a crossover from the hole pockets to the 'large' Fermi surface at $T^{*}$, which picture would nicely reproduce the pseudogap phenomenology observed [3] in cuprate superconductors.

Financial support of R. E. by the European Community and of Y. O. by the Saneyoshi Foundation and a Grantin-Aid for Scientific Reserach from the Ministry of Education, Science and Culture of Japan is most gratefully acknowledged.

FIG. 1. Photoemission spectra at half-filling for different values of $J / t$. The thin lines mark the spinon and holon band.

FIG. 2. Photoemission spectra at half-filling in the 12-site ring. For all $J / t$ the energy of the state marked by the black dot is taken as the zero of energy.

FIG. 3. (a) Schematic building principle of the spectral function at half-filling: Spinon band (thick dashed lines), and holon bands (thin full lines). (b) Theoretical spectrum overlaid with the numerical result for the 20 -site ring with $J / t=10$. The centers of the circles give the excitation energies and their diameter the pole strength of the peaks in the electron removal spectrum.

FIG. 4. Allowed momenta for the 20-site cluster.

FIG. 5. Photoemission spectra at half-filling for the $2 \mathrm{D}$ 20 -site cluster. For all $J / t$ the zero of energy has been set to the energy of the peak marked by the black dot.

FIG. 6. Photoemission spectra for the 12-site ring with 2 holes.

FIG. 7. Photoemission spectra for the 2D 18-site cluster with 2 holes. The spectra for momenta marked by an asterisk have been multiplied by a factor of 2 for clarity.

FIG. 8. Photoemission spectra for the 2D 20-site cluster with 2 holes. The spectra for momenta marked by an asterisk have been multiplied by a factor of 2 for clarity.

FIG. 9. Schematic representation of the 'band structure' in $1 \mathrm{D}$ and $2 \mathrm{D}$ systems as deduced from the diagonalization spectra.

FIG. 10. Left hand panel: Fermi surface for the Hamiltonian (1) with $t^{\prime} / t=0.3$, the full line corresponds to the main band, the dashed line to the shadow band. Right hand panel: Fermi surface for $t^{\prime} / t=0.3, \Delta / t=0.2$. 
[1] P. Aebi et al., Phys. Rev. Lett. 72, 2757 (1995).

[2] S. La Rosa et al., preprint.

[3] A. G. Loeser et al., Science 273, 325 (1996).

[4] D. S. Marshall et al., Phys. Rev. Lett. 76, 4841 (1996).

[5] C. Kim et al., Phys. Rev. Lett. 77, 4054 (1996).

[6] E. Dagotto, Rev. Mod. Phys. 66, 763 (1994).

[7] P.A. Bares and M. Blatter, Phys. Rev. Lett. 64, 2567 (1990).

[8] M. Ogata and H. Shiba, Phys. Rev. B 41, 2326 (1990).

[9] S. Sorella and A. Parola, J. Phys. Cond. Mat. 4, 3589 (1992).

[10] K. Penc, K. Hallberg, and H. Shiba, Phys. Rev. Lett. 77, 1390 (1996).

[11] J. Favand et al., e-print cond-mat 9611223.

[12] R. Eder et al., unpublished.

[13] R. Eder, Y. Ohta, and S. Maekawa, Phys. Rev. Lett. 74, 5124 (1995).

[14] R. Eder, P. Wrobel, and Y. Ohta, Phys. Rev. B 54, R11034 (1996).

[15] E. Dagotto and J. R. Schrieffer, Phys. Rev. B 43, 8705 (1990).

[16] R. Eder and Y. Ohta, Phys. Rev. B 50, 10043 (1994).

[17] J. Riera and E. Dagotto, unpublished.

[18] J. R. Schrieffer, X. G. Wen, and S. C. Zhang, Phys. Rev. B 39, 11663 (1989).

[19] S. A. Trugman, Phys. Rev. B 37, 1597 (1987).

[20] R. Eder and K. W. Becker, Z. Phys. B. 78, 219 (1990); see also M. Vojta and K. W. Becker, Phys. Rev. B 54, 15483 (1996).

[21] L. N. Bulaevskii, E. L. Nagaev, and D. I. Khomskii, Sov. Phys. JETP 27, 836 (1968).

[22] R. Eder, Y. Ohta, and T. Shimozato, Phys. Rev. B 50, 3350 (1994).

[23] A. Chubukov, Phys. Rev. B 52, R3840 (1995). 


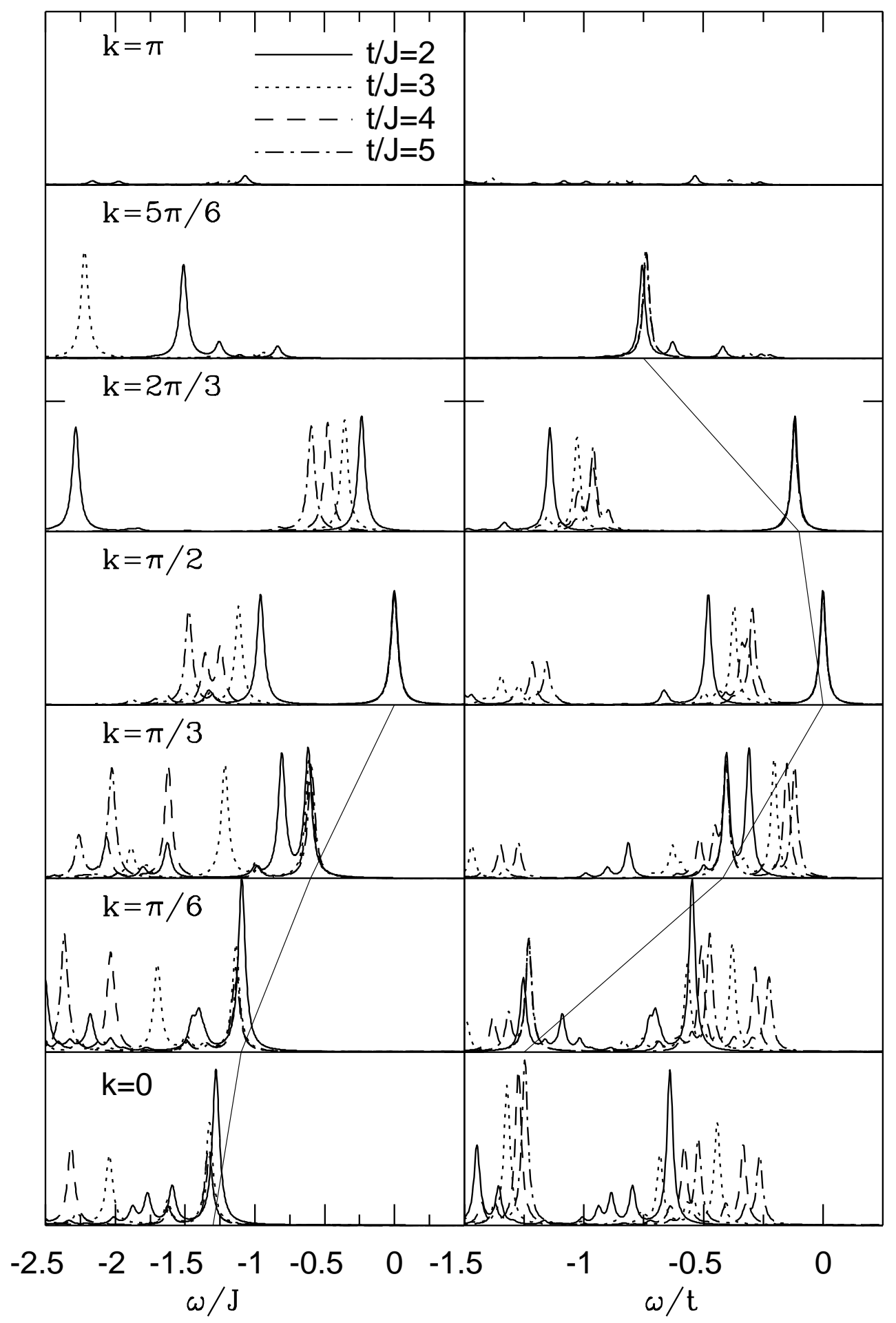




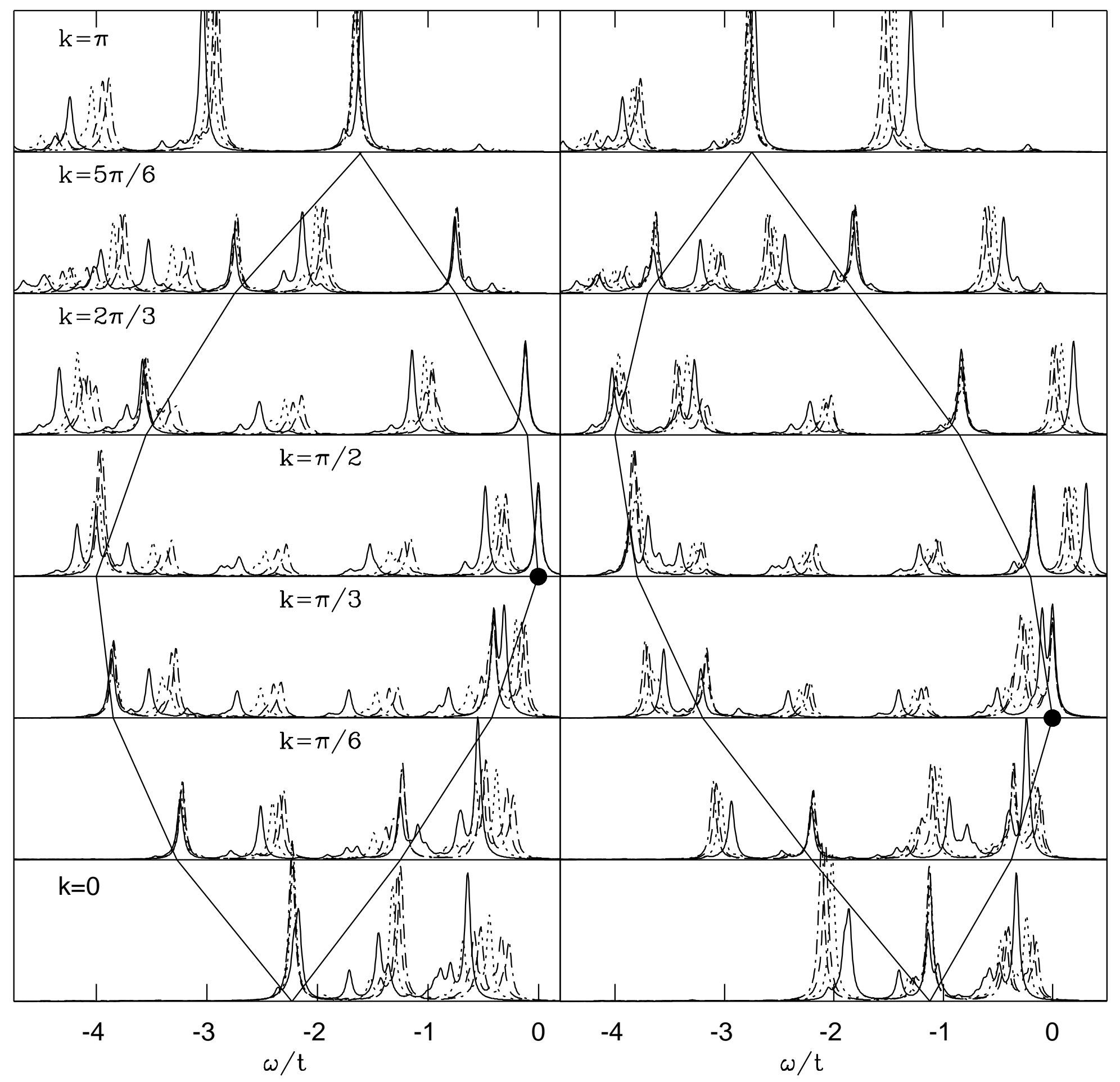



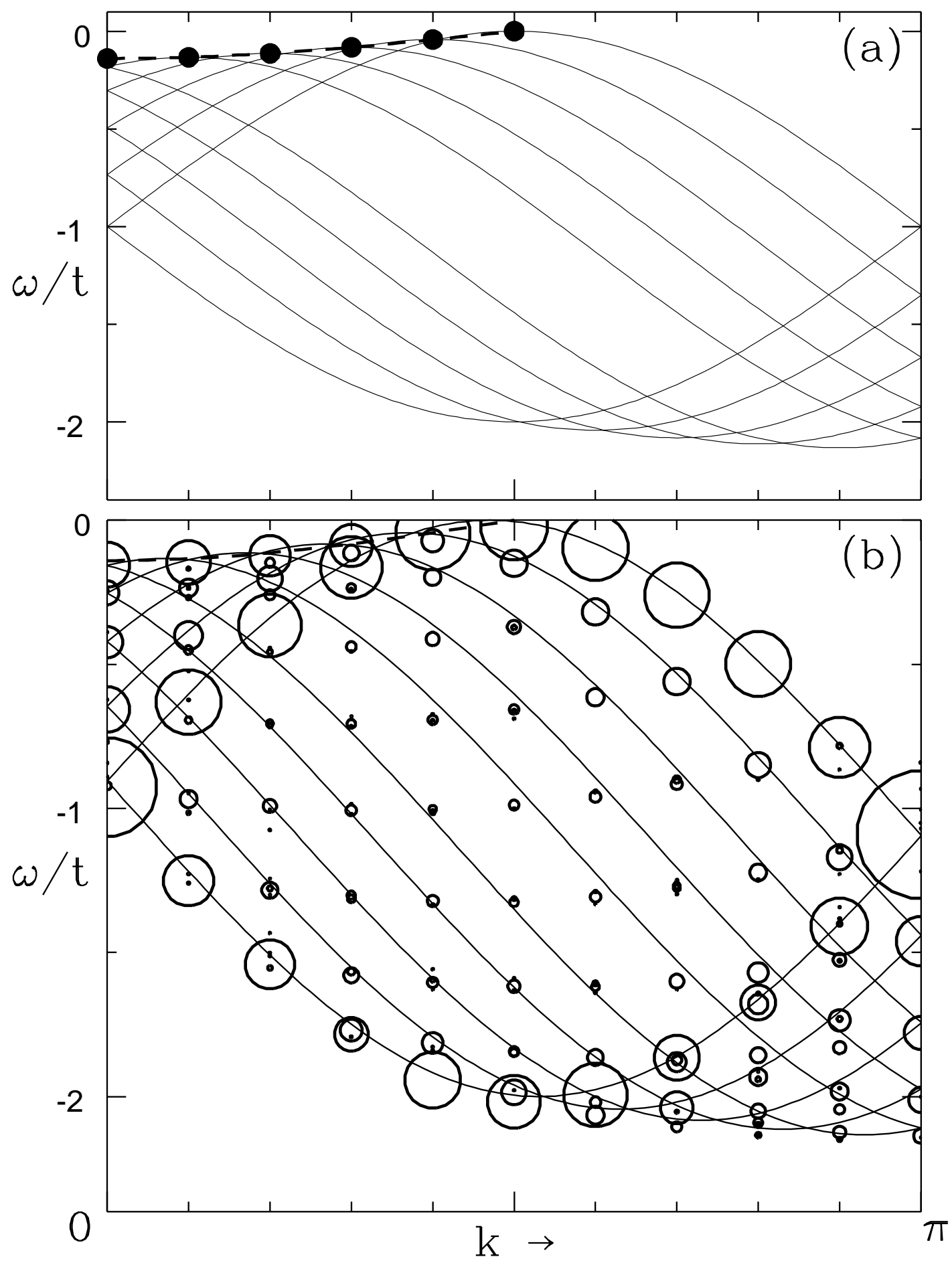


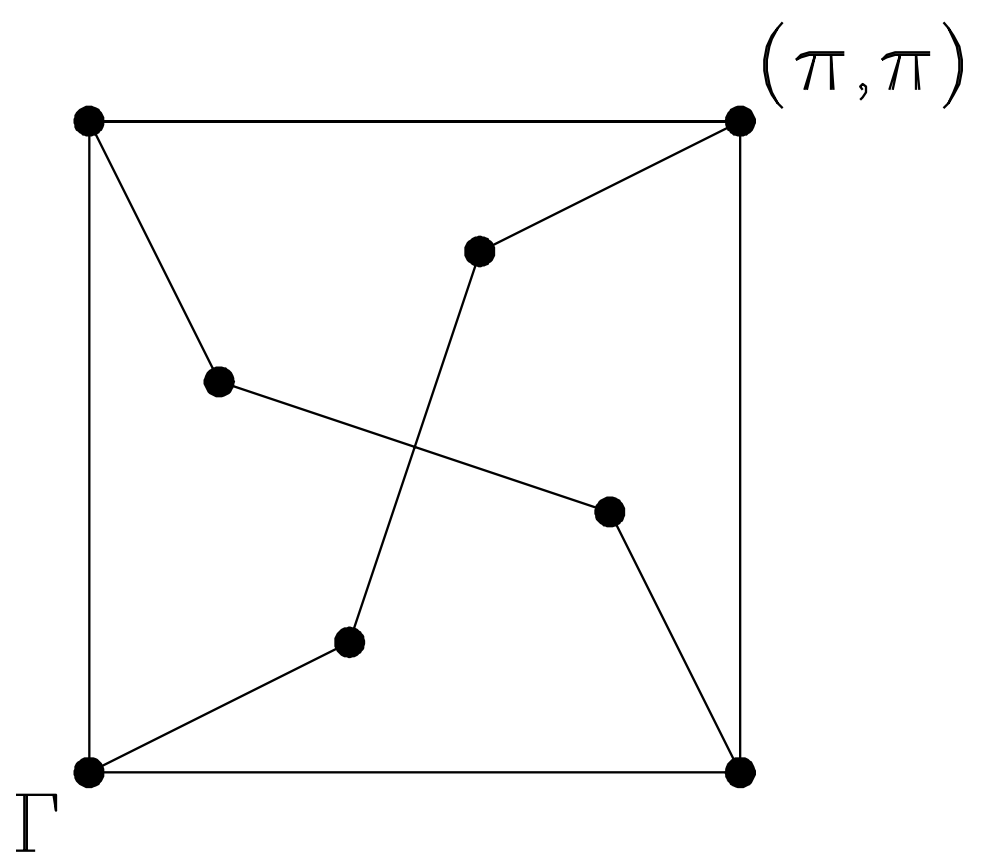




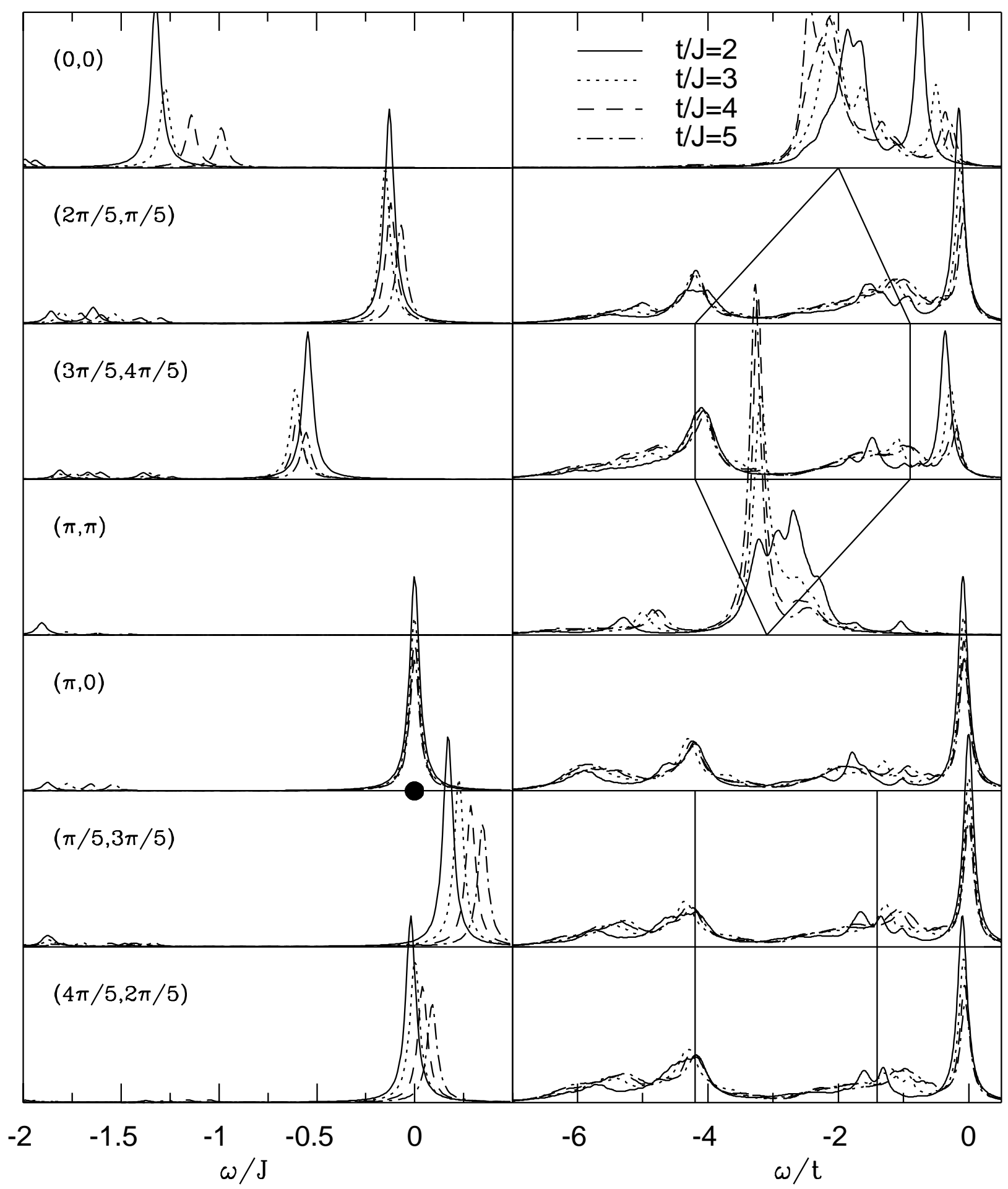




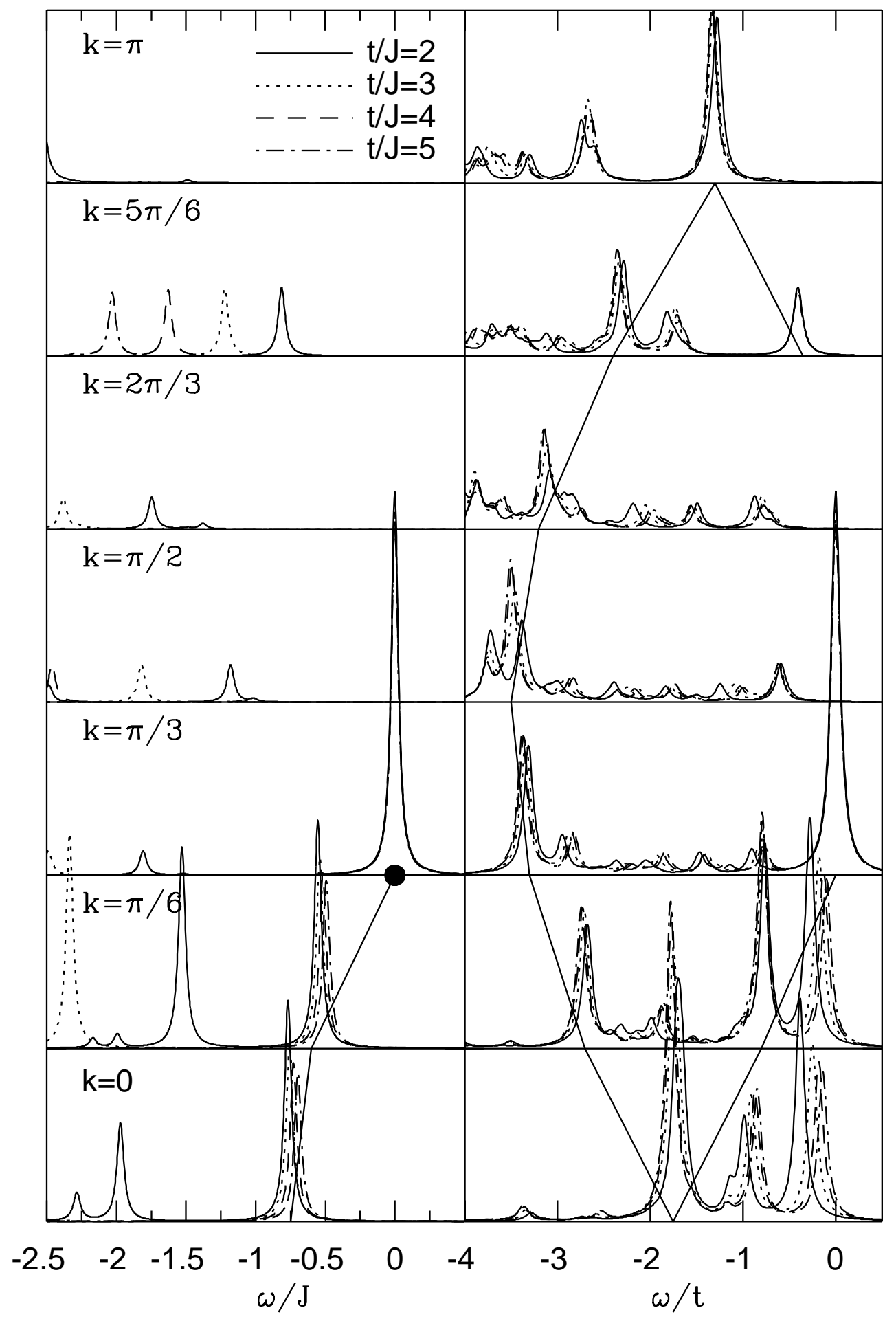




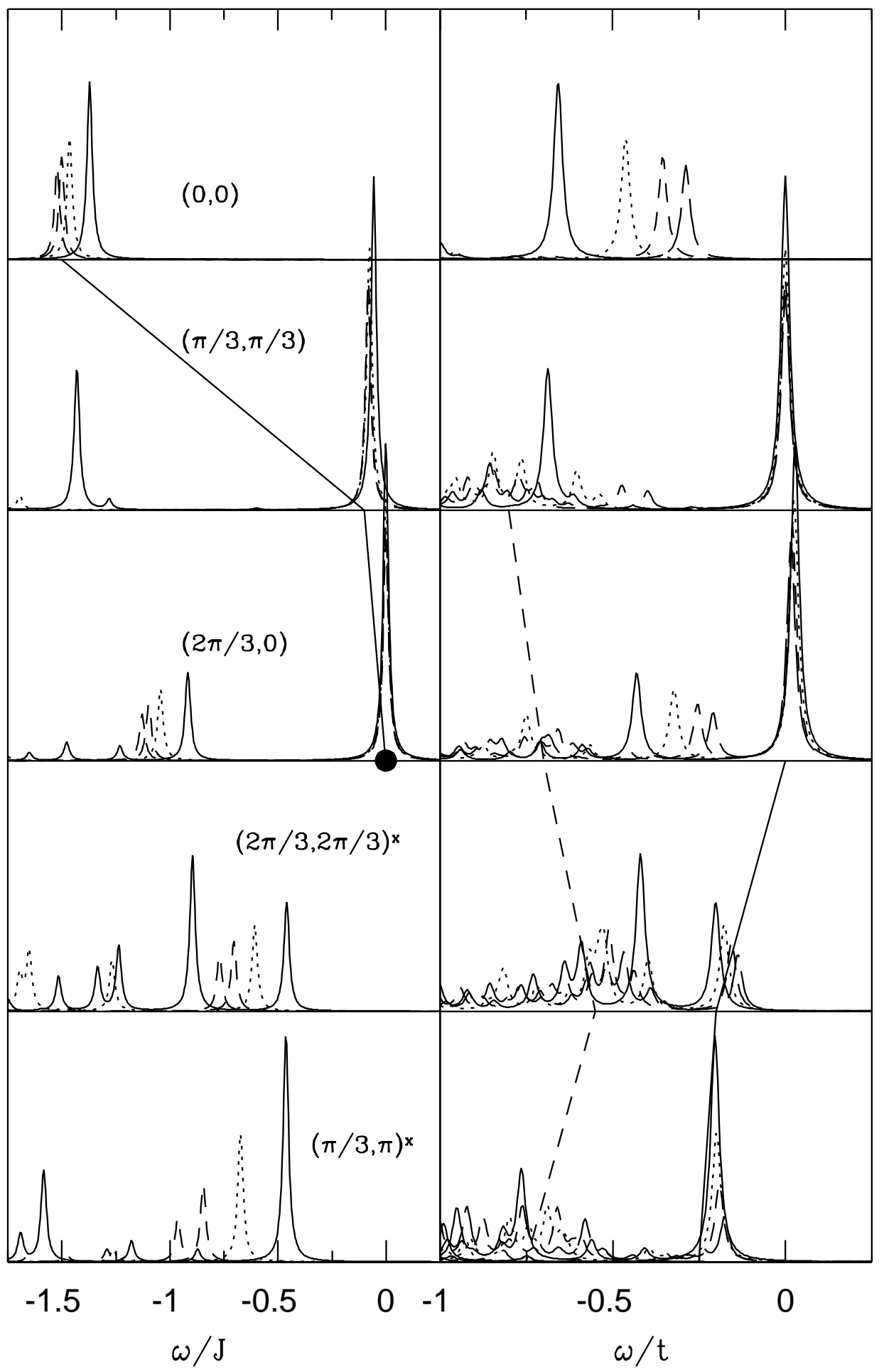




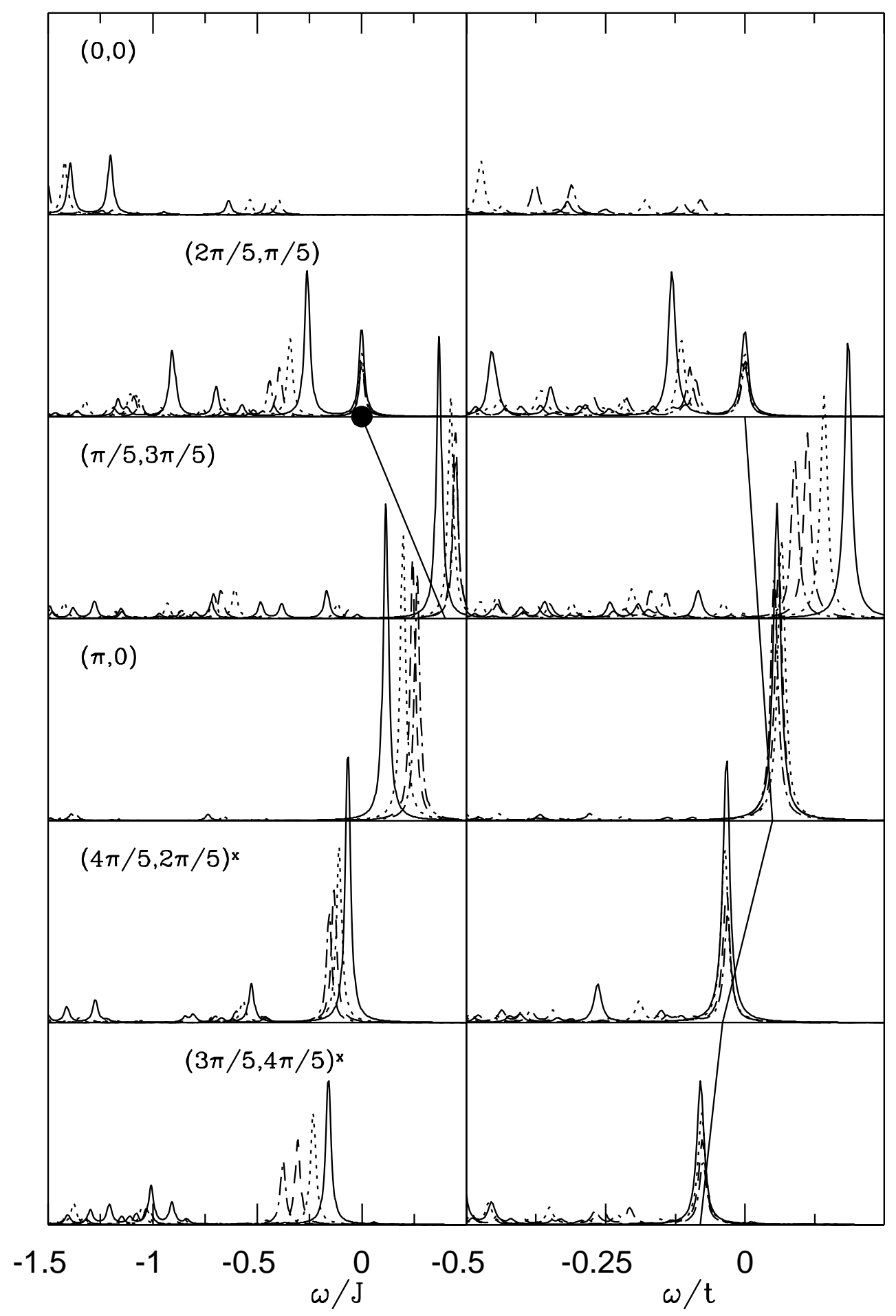




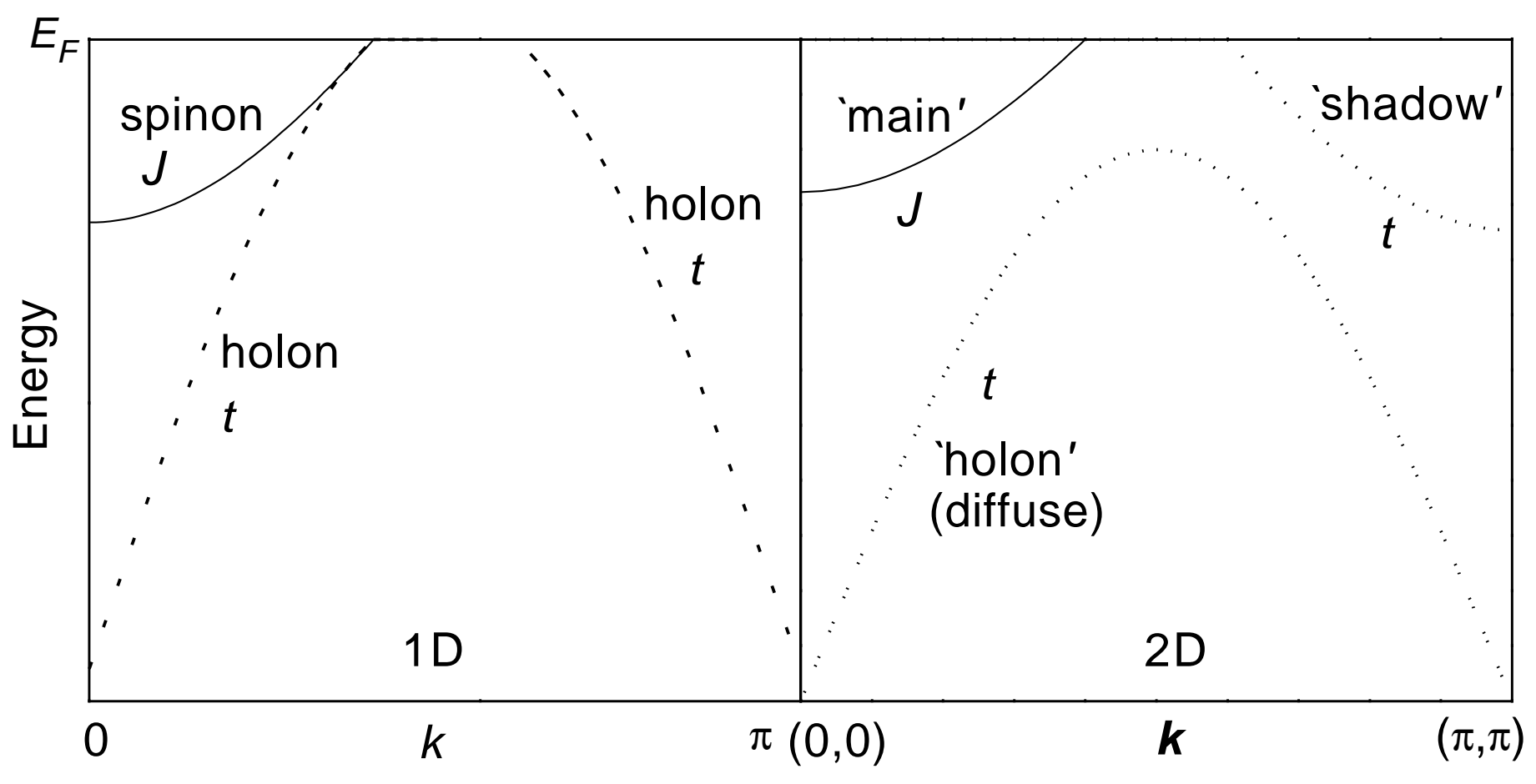

Figure 9 

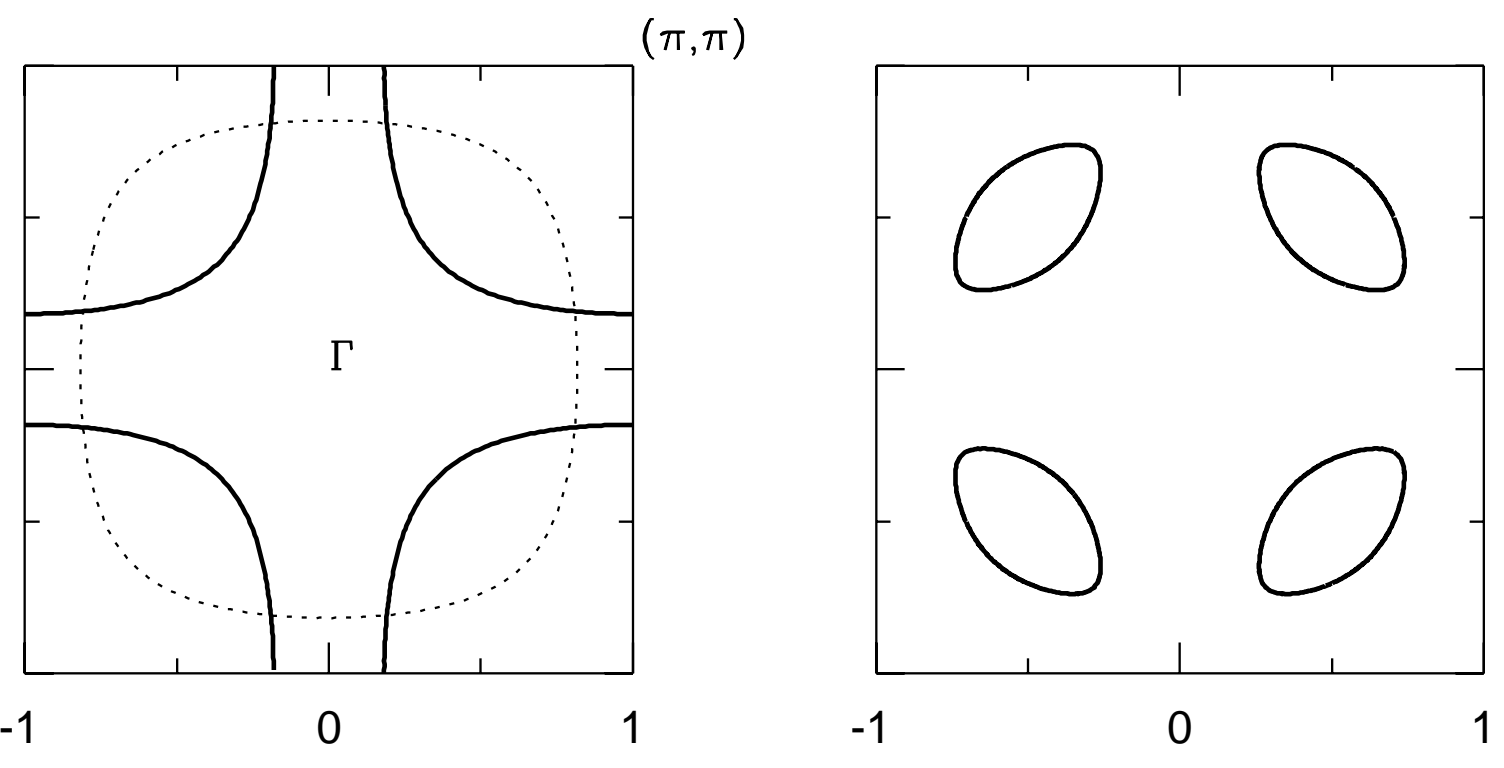